\title{
A systematic review of sub-microscopic Plasmodium vivax infection
}

\author{
Clarissa M. Moreira ${ }^{1,2^{*}}$, Mahmoud Abo-Shehada ${ }^{3}$, Ric N. Price ${ }^{2,4}$ and Chris J. Drakeley ${ }^{5}$
}

\begin{abstract}
Background: An accurate estimate of Plasmodium vivax prevalence is essential for the successful implementation of malaria control and elimination programmes. Prevalence estimates both inform control strategies and are used in their evaluation. Light microscopy is the main method for detecting Plasmodium parasitaemia in the peripheral blood, but compared to molecular diagnostics, such as polymerase chain reaction (PCR), has limited sensitivity.

Methods: A systematic review and meta-analysis was conducted to assess the effect of detection method on the prevalence of $P$. vivax and to quantify the extent to which P. vivax infections are undetected by microscopy. Embase, Medline and the Cochrane Database were searched for studies reporting prevalence by PCR and by microscopy and that contained all of the following key words: vivax, PCR, and malaria. Prevalence estimates and study meta-data were extracted systematically from each publication. Combined microscopy:PCR prevalence ratios were estimated by random effects meta-analysis. Sensitivity and specificity of microscopy were calculated using PCR as the gold standard.

Results: Of 874 studies reviewed, 40 met the criteria for inclusion contributing 54 prevalence pairs. The prevalence of $P$. vivax infection measured by PCR was consistently higher than the prevalence measured by microscopy with sub-patent parasitaemia. The mean prevalence of infection detected by microscopy was $67 \%$ (95\% Cl 59-73\%) lower than the prevalence detected by PCR. The detection of sub-patent parasitaemia did not vary according to the microscopy method (thick or, thick and thin smears), the PCR prevalence (as a measure of the true P. vivax prevalence), the type of blood used or DNA extraction method.

Conclusions: Quantifying P. vivax parasitaemia by PCR rather than microscopy consistently increased prevalence estimates by a factor of 2.3. Whilst the sensitivity of microscopy can be improved by better methods, molecular methods have potential to be scaled up to improve the detection of $P$. vivax transmission reservoirs.
\end{abstract}

Keywords: Malaria, Plasmodium vivax, Polymerase chain reaction, Microscopy, Diagnostics, Prevalence

\section{Background}

Almost half of the world's population is at risk of infection with Plasmodium vivax [1]. Most cases originate in South East Asia and the Western Pacific and a significant number occur in South America and the Horn of Africa $[1,2]$. P. vivax is associated with substantial morbidity and severe and fatal disease in endemic countries [3-6]. $P$ vivax infection is characterized by comparatively low parasitaemia compared to Plasmodium falciparum, and in co-endemic regions vivax parasitaemia is often conservatively documented as $P$. falciparum reducing the

\footnotetext{
*Correspondence: clarissamoreira1@gmail.com

1 WorldWide Antimalarial Resistance Network (WWARN), Oxford, UK

Full list of author information is available at the end of the article
}

reported prevalence [3]. Many nations evaluating their prospects for malaria elimination are endemic for $P$. vivax and as successful control programmes reduce the risk of $P$. falciparum the relative proportion of $P$. vivax infection rises. Accurate data on $P$. vivax prevalence and transmission patterns is important for the progress of elimination campaigns and focusing malaria control efforts [4].

Light microscopy examination of blood films is the main method for detecting peripheral parasitaemia and for differentiation of Plasmodium species [5]. The World Health Organization (WHO) guidelines for malaria diagnosis and treatment recommend that malaria treatment be given only after a positive parasitological test result from either microscopy or a 
rapid diagnostic test (RDT) [6]. The advantages of light microscopy make it an ideal diagnosis tool in resource poor settings however its accuracy depends upon the technician's skill level and can be adversely affected by operational constraints or technical problems. Lowdensity infections often remain undetected by microscopy $[7,8]$. As $P$. vivax is characterized by low level parasitaemia microscopy may not be the most appropriate tool for accurate diagnosis. Molecular methods, e.g. polymerase chain reaction (PCR), depend on DNA amplification approaches and have higher sensitivity than microscopy [9]. Despite the greater sensitivity of PCR it is not widely used due to the lack of a standardized methodology, high costs, and the need for highly-trained staff. PCR is increasingly used in epidemiological studies, but rarely used in routine clinical diagnosis.

Most $P$. vivax cases occur in low transmission settings where asymptomatic carriage occurs relatively frequently [10]. With the reliance on symptoms driving the presentation of infected patients and microscopy used as the main diagnosis tool, asymptomatic and sub-microscopic infections are likely to remain undetected and untreated in vivax endemic populations. Sub-microscopic infections of $P$. falciparum have been shown to infect mosquitoes and transmit malaria [11]. Sub-microscopic $P$. vivax infections may similarly contribute to the infectious reservoir and maintain transmission. A review on asymptomatic malaria in Brazil highlighted the importance of accurate diagnosis and detection of all Plasmodium cases for the malaria control programmes to be effective [7]. A prevalence ratio reported from a study in Peru indicates that $78 \%$ of infections would go undetected if microscopy alone was used in surveillance programmes [8]. In a study from Iran microscopy did not detect any vivax infection in the 900 samples, whereas PCR detected ten positive samples out of 871 [12].

In a review exploring the proportion of sub-microscopic $P$. falciparum infections, Okell et al. documented reduced sensitivity of microscopy which detected on average $50 \%$ of falciparum infections detected by PCR [13]. Cheng et al. recently reviewed 25 publications (31 surveys) from 1996 to 2010 reporting a mean of $69.5 \%$ sub-microscopic $P$. vivax infections [14]. The current review extends the work of Cheng et al. with a meta-analysis and the inclusion of additional studies; since 2010, a further 11 prevalence studies reported microscopy and PCR P. vivax prevalence and an additional seven were identified from 1996 to 2010. The aim was to re-quantify the extent of sub-microscopic $P$. vivax infections detected by PCR and identify whether this ratio varies with transmission intensity, location of study or laboratory methods.

\section{Methods}

This systematic review used a predefined protocol and followed the PRISMA guidelines [15]. PubMed, EMBASE and the Cochrane Library were searched using MeSH search terms and Boolean operators: "malaria" AND "PCR" AND "vivax" up to the 15 September 2014. The search was restricted to English language publications with no time limits. Results of each search were exported and duplicates removed. This high-yield search strategy was used to ensure capture of all relevant articles. Titles and abstracts of all articles were initially scanned to identify prevalence studies. Case reports, case series, efficacy studies, entomological surveys, non-human studies, immunological studies, genetic sequencing studies and other non-relevant articles were excluded. Full texts of articles identified as potentially relevant in this initial screen were assessed against the full eligibility criteria using a standardized form. References of relevant articles were scanned to identify additional studies.

\section{Selection criteria}

Eligible studies reported the prevalence of $P$. vivax by microscopy and PCR in the same population living in a malaria endemic country. If only a subset of participants were tested by microscopy or PCR, the study was included providing the subset was selected randomly. Exclusion criteria included: studies of imported malaria, studies where a large-scale intervention was implemented before the measurement of prevalence (e.g. insecticide-treated bed nets or mass drug administration), studies in pregnant women, studies that select participants based on parasitaemia levels, malaria symptoms or malaria diagnosis, studies of a population not representative of a defined endemic area (e.g. studies in refugees or migrant workers), studies with less than 20 blood samples tested by either method, and studies where no P. vivax was detected by PCR or microscopy. When the presence of malaria symptoms was not specifically stated it was assumed that participants seeking treatment or care at health facilities were symptomatic and these studies were excluded. In cohort studies, data were extracted from the baseline observation only.

\section{Data extraction}

From each eligible study the following information was extracted: month and year of sample collection, location, age criteria for inclusion, age range of included participants, prevalence of $P$. vivax malaria by microscopy, prevalence of $P$. vivax malaria by PCR, number of falsepositives (i.e. number of samples microscopically positive and PCR negative) and number of false-negatives (i.e. number of samples microscopically negative and PCR positive) and PCR and microscopy methodology. 
Authors were not contacted for further information and no studies were excluded on the basis of quality.

\section{Statistical analysis}

The parasite prevalence by microscopy and PCR were calculated for each study, once for $P$. vivax monoinfections and again for all $P$. vivax infections (i.e. infections where P. vivax was detected either alone or with any other Plasmodium species). Prevalence of infection detected by microscopy was compared to the prevalence of infection detected by PCR to produce a microscopy:PCR prevalence ratio and the sub-microscopic prevalence was calculated as: PCR prevalence minus the microscopy prevalence. PCR was considered the reference standard and the numbers of false-positives and false-negatives were used to calculate sensitivity and specificity estimates for microscopy when this information was available. Log transformed microscopy: PCR prevalence ratios and the sample size for each study were used to derive inversevariance weighted fixed effects and random effects metaanalysis combined estimates [16]. The Kruskal-Wallis method was used for nonparametric comparisons, and Student's $t$ test for parametric comparisons. For categorical variables percentages and corresponding $95 \%$ confidence intervals (95\% CI) were calculated using Wilson's method. Proportions were examined using $\chi^{2}$ with Yates correction or by Fisher's exact test. The Chi squared heterogeneity statistic was used to assess the between-study heterogeneity. Forest plots and combined random-effects estimates were produced for sub groups (e.g. microscopy method) to determine if heterogeneity was accounted for by any methodological differences. All analyses were performed using Stata software (11.0; StataCorp).

\section{Results}

\section{Literature search}

A search of Pubmed, EMBASE and the Cochrane Library identified 874 unique publications; 182 were selected for full text evaluation. Forty met all eligibility criteria (Fig. 1) $[8,12,17-54]$. Details for the exclusion of full text articles are provided in Additional file 1. The most common reasons for exclusion were surveys of patients with malaria or malaria symptoms $(\mathrm{N}=84)$, study was not population based $(\mathrm{N}=24)$, prevalence from either method was not reported $(\mathrm{N}=17)$ and samples chosen for PCR were not randomly selected $(\mathrm{N}=8)$. Five studies were excluded since no $P$. vivax parasites were detected either by PCR or by microscopy [55-59].

\section{Description of included studies}

Most of the 40 included studies were derived from crosssectional surveys $(\mathrm{N}=31,78 \%)$, three were cohort studies $[23,47,60]$ and three studies recruited participants via active case detection [30, 44, 46]. Three studies did not report the study design $[23,51,61]$.

The sampling strategy was reported for 27 studies (68\%). In 14 (52\%) of these studies, all households were visited and informed consent sought from all eligible residents. Five studies reported random sampling of individuals, two studies reported random sampling at the household level, one reported random sampling of schools where all students at the selected schools were included and one study reported multistage cluster sample with random cluster sampling of villages then random household sampling. Two studies reported convenience sampling (one of which provided no further information [36]) and the other took blood samples from all neighbours of confirmed malaria cases admitted to hospital [20]. Two studies reported active case detection, one at mobile malaria clinics [26] and the other through contact tracing of confirmed malaria cases and high risk group screening [42].

The 40 publications yielded 54 pairs of microscopy and PCR prevalence estimates. In 14 studies prevalence was reported for different localities which were analysed as independent observations. Two studies compared microscopy to two different PCR methods contributing two paired prevalence estimates [47, 62]. Key study features are highlighted in Additional file 2. Most prevalence estimates were from Papua New Guinea $(\mathrm{N}=13)$ and Brazil $(\mathrm{N}=13)$. Only two prevalence pairs were from Africa and one from the Middle East. Blood samples were collected between 1996 and 2012, with the majority collected after $2004(\mathrm{~N}=34)$. Eight studies did not report the year of sample collection. Most blood samples were from participants of all ages, five studies were restricted to children only (maximum age 3 or 5 years or age limited not reported), two restricted to adults aged more than 15 years and four studies did not report the age of participants (see Additional file 2).

In $73 \%(29 / 40)$ of studies all blood samples were assessed by both microscopy and PCR. In five studies more than $90 \%$ of microscopy samples were assessed by PCR [17, 21, 23, 28, 37]. For three prevalence estimates, 8,19 and $34 \%$ of microscopy samples were randomly selected for molecular testing [36, 37]. One study tested $85 \%$ of microscopy samples by PCR but did not report how these samples were chosen [20], another tested $80 \%$ of samples by PCR due to a reported difficulty in collecting whole blood samples from children less than 5 years [8]. Another study selected all samples from villages where there was any microscopy-positive sample, plus a $10 \%$ random selection of microscopy-negative samples from all villages for testing by PCR [18]. In this last case, the majority of samples tested by PCR were not samples that were confirmed microscopy-positive (only 8 samples 


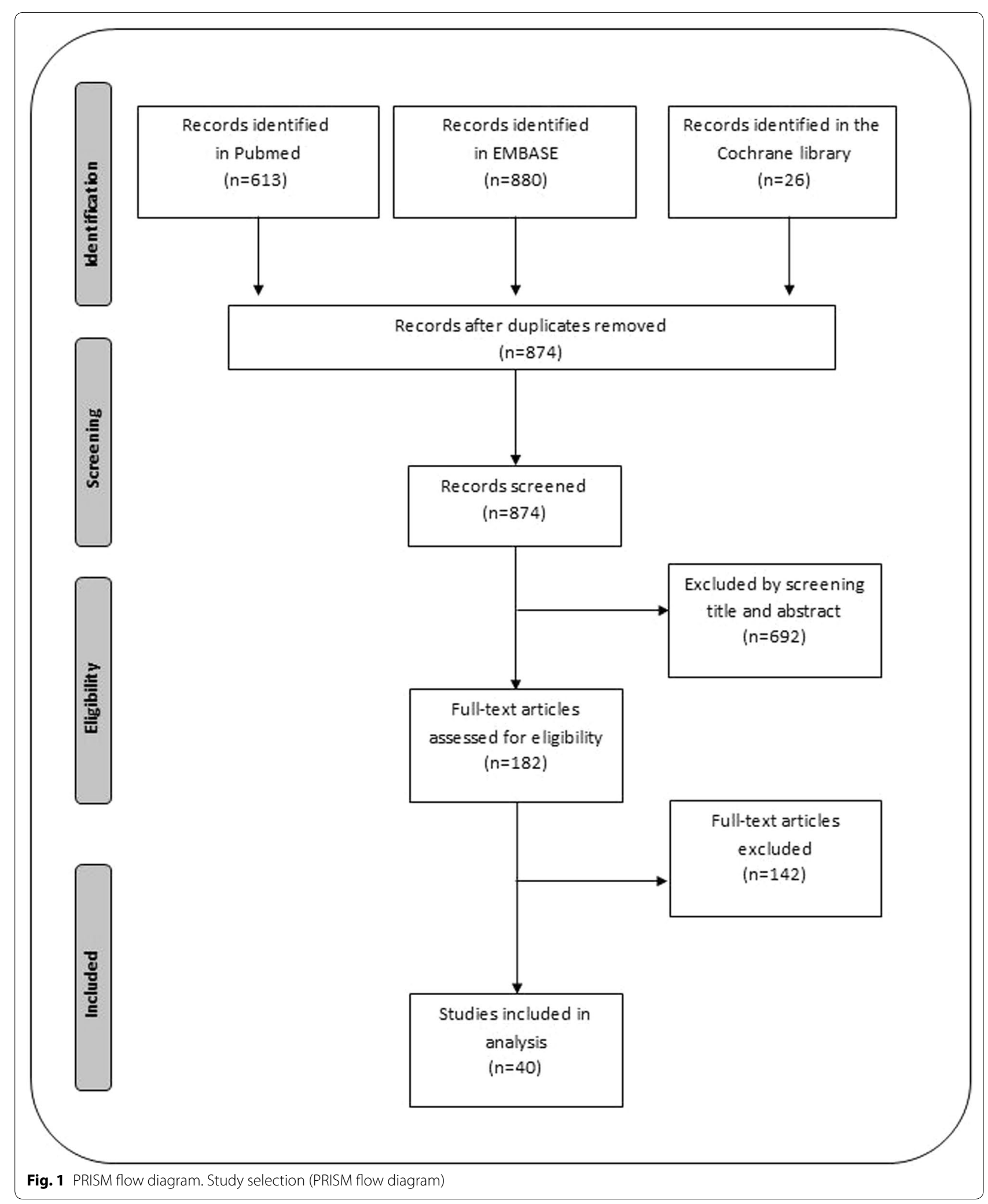


out of 3223 tested by PCR were chosen because they were positive by microscopy), so this study was included. The final study selected $7 \%$ of microscopy samples that were chosen to be representative of the age, gender and village composition for testing by PCR [61].

The overall median sample size was 504 (range 79-16,229) for microscopy and 482 (range 79-8590) for PCR. Most microscopy measurements used thick and thin smears with Giemsa staining $(\mathrm{N}=28)$, the remaining studies $(\mathrm{N}=12)$ used only thick smears with Giemsa staining. The most referenced PCR method was nested PCR using the protocol of Snounou et al. $(\mathrm{N}=18)$ [9]. Other reported PCR methods include the semi-quantitative PCR (LDR-FMA) of McNamara, the semi-nested multiplex PCR of Rubio, various real-time PCR methods $[22,45,62,63]$ and one article reported using a newly designed mitochondrial-DNA-based PCR method [28].

All studies described the type of blood and DNA extraction method used for PCR analysis. Twenty studies (50 \%) used whole blood (primarily $>200 \mu \mathrm{L}, \mathrm{N}=15$ ), 17 (42.5\%) used blood from filter paper, and three studies (7.5\%) reported using both blood from filter paper and whole blood for DNA extraction. Two of the three studies that reported both methods used filter paper for some surveys and whole blood for others and the other study used whole blood for participants aged $>5$ years and filter paper for participants $<5$ years.

\section{Quality assessment}

Only four studies reported blinding of technicians to results of the other detection method (i.e. microscopists blinded to PCR results and vice versa). Double reading of microscopy slides was reported for 40 prevalence pairs (74\%). Many studies reported the use of 'expert' microscopists and specified their training, years of experience or results when given a blinded test before the study started. Only seven studies reported blinding of the second microscopists to the results of the first, most did not report if this occurred $(\mathrm{N}=30)$. Nineteen studies $(48 \%)$ reported the use of negative controls during PCR.

\section{Prevalence measurements}

The prevalence of $P$. vivax infection measured by PCR was consistently higher than the prevalence measured by microscopy (Fig. 2). One study reported a prevalence by microscopy higher than the prevalence by PCR [39]. The mean prevalence by PCR was $18 \%$ (95 \% CI 13.3-23.4) and the mean prevalence by microscopy was $7.8 \%$ (95 \% CI 4.4-11.1). Forty-two of the 54 prevalence pairs reported the prevalence of $P$. vivax monoinfections (infection with $P$. vivax only) separately from the prevalence of all $P$. vivax infections (mono or mixed infections). The variation in prevalence was greater when including

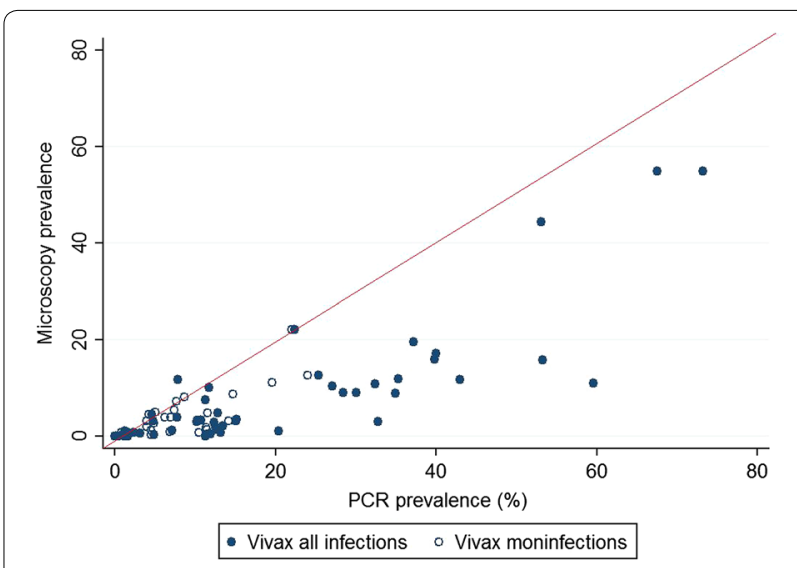

Fig. 2 PCR P. vivax prevalence versus microscopy $P$. vivax prevalence, monoinfections and mixed infections. Scatter plot of the prevalence of $P$. vivax infection as determined by $P C R$ versus prevalence of $P$. vivax as detected by microscopy. Prevalence pairs including only monoinfections (infections with only P. vivax) are shown in open circles and the prevalence pairs including all infections ( $P$. vivax monoinfections and $P$. vivax infections where another species is also detected) are shown in filled circles. The line shows the expected association if both techniques were equally sensitive

mixed infections compared to including monoinfections only, i.e. concordance between the two techniques was greater when considering monoinfections only (Fig. 2). PCR detected mixed infections in $1.8 \%$ of parasitaemias compared to $0.65 \%$ detected by microscopy. For nine prevalence pairs, $P$. vivax parasitaemia was not detected by microscopy but was detected by PCR (PCR prevalence range $0.18-11.2 \%$ ). The prevalence of sub-microscopic $P$. vivax ranged from 0.01 to $48.8 \%$ and was higher in the Asia Pacific region [mean $=18 \%$ (range 0.32-48.8 \%)] compared to South America [9.4 \% (range 0.34-31.3\%); $\mathrm{p}=0.02]$ and Asia [1.7 \% (range 0.01-29.8\%); $\mathrm{p}=0.001]$. There was no statistical difference in the prevalence of sub-microscopic $P$. vivax between Asia and South America ( $\mathrm{p}=0.18$; Fig. 3$)$.

\section{Prevalence ratios}

Prevalence ratios were calculated for $83 \%$ (45/54) of the prevalence pairs; in the remaining nine pairs no $P$. vivax was detected by microscopy precluding derivation of a ratio. A forest plot of the prevalence ratios with $95 \%$ confidence intervals is shown in Fig. 4. The prevalence ratios were highly heterogeneous between studies and ranged from 0.03 to 1.49 (heterogeneity Chi squared $=936.51$, $\mathrm{p}<0.001$, Fig. 4). The combined microscopy: PCR prevalence ratio estimate using random effects at the study level was 0.33 (95\% CI 0.27-0.41), i.e. the prevalence of infection detected by microscopy was $67 \%$ lower than the prevalence of infection detected by PCR. The corresponding figure when considering monoinfections only, 


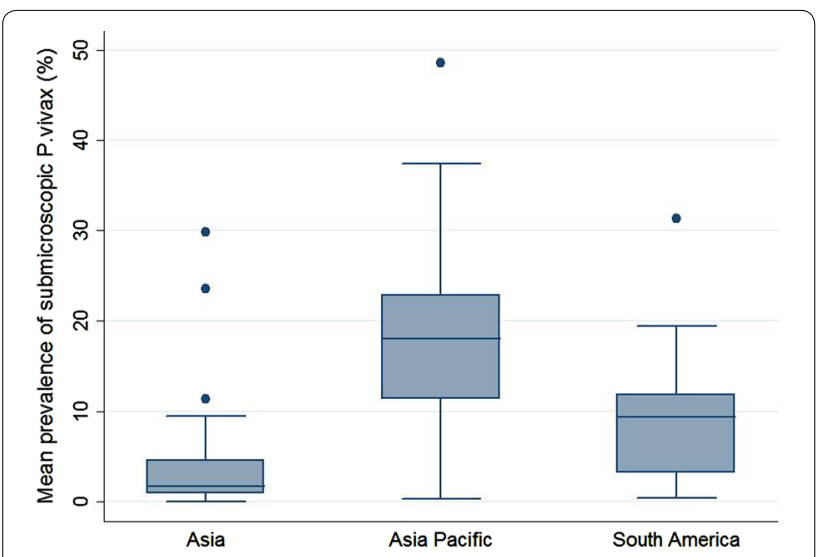

Fig. 3 Sub-microscopic P. vivax prevalence by region. Box plots showing the median and the IQR of sub-microscopic $P$. vivax prevalence by region (Asia Thailand, Sri Lanka, Lao PDR, Vietnam, Malaysia and Cambodia; Asia Pacific Indonesia and Papua New Guinea; South America Brazil, Venezuela and Peru)

was 0.37 (95\% CI 0.29-0.48). Overall the prevalence of infection detected by microscopy was $60 \%$ (95\% CI 36-75\%) lower than the prevalence of infection detected by PCR in Asia, $67 \%$ (95\% CI 57-75\%) lower in the Asia Pacific and $72 \%$ (95 \% CI 58-81 \%) lower in South America. Studies from South America were less heterogeneous (heterogeneity Chi squared $=88.03$ ) compared to Asia (185.07) and Asia Pacific (88.03).

The combined prevalence ratio estimates did not vary between studies that used thick and thin smears for microscopy and those that used thick smears only. Likewise the prevalence ratio did not vary between studies that used filter paper for DNA extraction compared to those that used whole blood. However the majority of prevalence pairs $(14 / 23)$ that used whole blood were from Asia, whereas prevalence pairs that reported filter paper for DNA extraction were from the Asia Pacific region $(14 / 28)$ or South America (10/28). Reporting the use of a negative control was not associated with a significant difference in the combined microscopy: PCR prevalence ratio: 0.36 (95\% CI 0.27-0.47) versus 0.31 (95 \% CI 0.23$0.42),(\mathrm{p}=0.24)$. Prevalence ratios did not significantly vary between studies that used nested PCR compared to other PCR techniques $(\mathrm{p}=0.71)$. The microscopy: PCR prevalence ratio showed a weak positive association with the underlying PCR prevalence, increasing by 0.047 (95\% CI 0.002-0.09) per $10 \%$ increase in PCR prevalence (Fig. 5).

\section{Sensitivity and specificity}

The sensitivity and specificity of microscopy was calculated for 20 prevalence paired estimates. Sensitivity and specificity were 0 and $100 \%$ respectively for 7 prevalence pairs where no $P$. vivax parasites were detected by microscopy. Assuming that one sample was positive by both microscopy and PCR, and that one sample was positive by microscopy and negative by PCR allowed calculation of sensitivity and specificity for these seven studies; results are available in Additional file 3. Overall, microscopy was highly specific with less than $2 \%$ false positives. However the sensitivity varied from 4.2 to $94 \%$.

\section{Discussion}

Data from 40 published studies show that PCR detects $67 \%$ more $P$. vivax infections than microscopy in surveys of endemic populations. This result is similar to findings by Cheng et al. who reported on 25 studies in which $P$. vivax was not detected by microscopy in $69.5 \%$ of estimates. The additional 18 studies included in the current analysis generally reported higher prevalence by both microscopy and PCR; the mean PCR prevalence was $18.6 \%$ (range $0.18-73.2 \%$ ) compared to a range of PCR prevalence estimates of $0.2-59.5 \%$ reported by Cheng.

Both reviews are also consistent with a literature review of $P$. falciparum PCR and microscopy prevalence reported by Okell and colleagues in which microscopy detected $51 \%$ of all parasitaemias detected by PCR [13]. There are several characteristics of $P$. vivax infections that make diagnosis by microscopy more difficult than diagnosis of $P$. falciparum including the fact that $P$. vivax and $P$. falciparum are co-endemic in most vivax endemic areas and the detection of lower levels of $P$. vivax parasitaemia is difficult in the presence of higher levels of $P$. falciparum parasitaemia [3]. PCR has greater sensitivity to identify mixed infections [64], and this was apparent in the discrepancy between microscopy and PCR being greatest when considering all $P$. vivax infections (mono and mixed infections). Similar results are apparent in cross sectional surveys where PCR is as much as 30 -fold more efficient at detecting mixed infections than microscopy alone [17]. The difference between prevalence ratios when detecting monoinfection and prevalence ratios when detecting all $P$. vivax infections, showed a similar trend although this did not reach statistically significance likely due to the limited number of prevalence pairs.

Only ten studies reported sensitivity and specificity measurements and these studies specifically aimed to measure these parameters for either PCR or microscopy. The wide range of microscopy sensitivities mirrored published sensitivities although microscopy sensitivity $<50 \%$ is less frequently reported in the literature than observed here. The sensitivity and specificity of microscopy is highly dependent on slide quality and the skill of microscopists [65]. Unfortunately these factors could not be considered in this analysis; both are subjective and rarely reported. The low prevalence of vivax malaria 


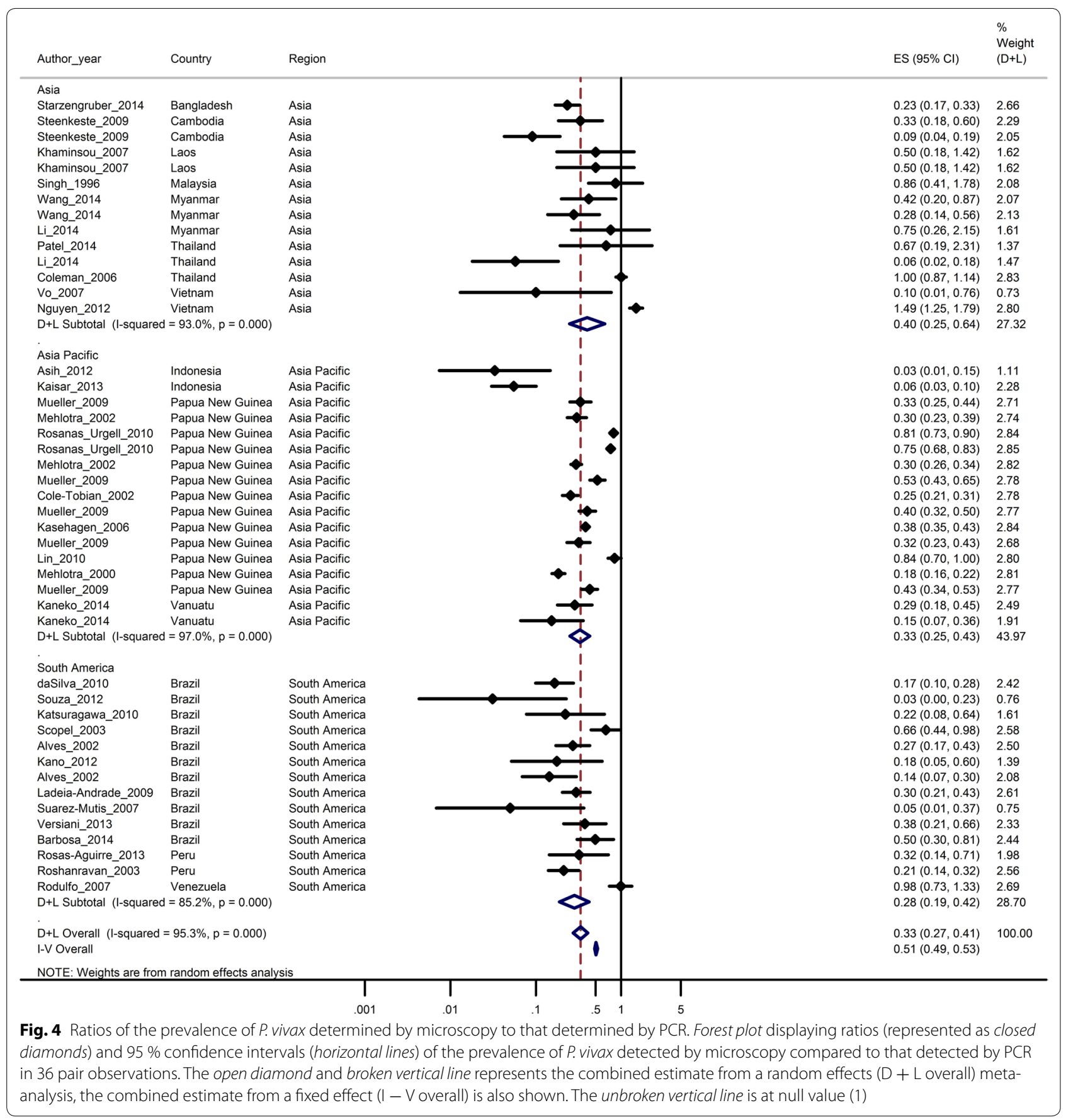

could explain the variability in sensitivity estimates as the number of positive results for both PCR and microscopy used in sensitivity calculations was small.

The studies included in this review are representative of the geographical distribution of $P$. vivax, with most but not all $P$. vivax endemic countries represented. The inclusion of only English publications introduced a bias towards studies from English speaking countries and a large number of potentially eligible publications from
Brazil were not available in English [66]. The analysis focused on blood samples representative of endemic populations with a high proportion of included studies adopting comprehensive sampling strategies in an attempt to determine covariates in endemic populations; however the detection of sub-microscopic infections in high-risk groups or other populations excluded from this review also have public health relevance that warrants investigation. 


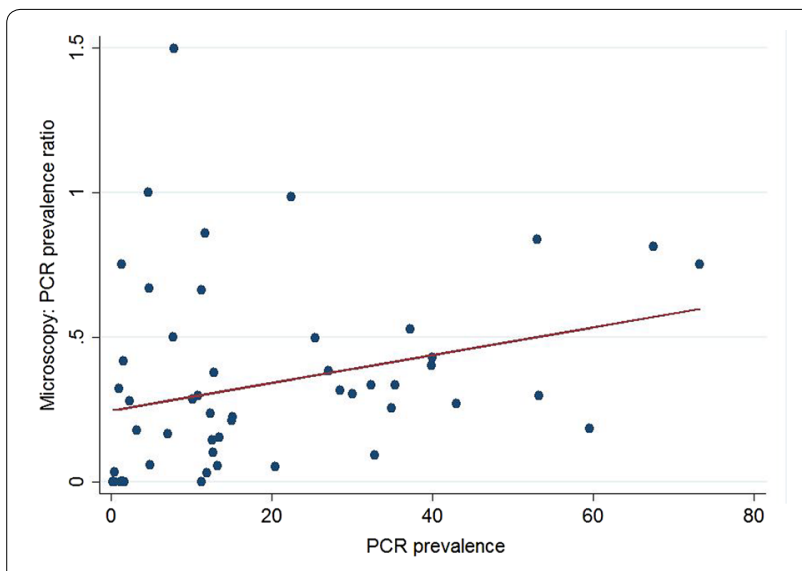

Fig. 5 Scatter plot of the microscopy: PCR prevalence ratio versus the underlying PCR prevalence

With only 54 paired prevalence estimates included in this meta-analysis, the power to detect key covariates was limited. Studies where patients were selected on the basis of malaria symptoms were excluded and this one criterion significantly reduced the number of studies available. Five studies were excluded as no $P$. vivax was detected by either technique, in addition, prevalence ratio could not be calculated for nine prevalence pairs when no $P$. vivax was detected by microscopy, the least sensitive detection method. Therefore, the extent to which microscopy failed to detect infections of $P$. vivax in low transmission areas is likely underestimated. Sample size is an important consideration in the design of studies where few infections are detected. The studies in this review that did not microscopically detect any $P$. vivax infections but did detect vivax infections with PCR were of varying samples sizes and two were large studies $(\mathrm{N}=3316, \mathrm{~N}=1527)$. This indicates that when only using microscopy, increasing the sample size alone will not allow detection of all existing $P$. vivax infections.

The detection limit of both techniques is dependent on the volume of blood examined. In theory, less blood is examined using standard microscopy techniques $(\sim 5 \mu \mathrm{L})$ than when a PCR assay is used [60, 67]. This meta-analysis did not consider the exact volume of blood examined since the amount of blood used for DNA extraction for PCR was reported in only $18 / 40$ studies and the amount of blood taken from participants reported in only 5/40 studies. Furthermore, nearly all studies that did report the amount of blood used for DNA extraction reported using $200 \mu \mathrm{L}$. The type of blood sample and DNA extraction method used for PCR analysis was examined and while the PCR prevalence was higher in studies that used whole blood versus studies that used filter paper this likely reflected regional variation and since the majority of studies using whole blood were from Asia and the majority of studies using filter paper being from the Asia Pacific or South America.

Future studies should examine the prevalence ratios of all malaria species in the same population to see if the sensitivity and specificity of microscopy and PCR varies according to the species examined. Although PCR is unlikely to be routinely used for screening in low-income countries, quantification of sub-microscopic infections under different transmission settings could be used to accurately estimate the true prevalence of $P$. vivax infections. Mathematical models could predict the extent of sub microscopic infections given a number of key parameters on the population characteristics and the transmission setting. Bayesian approaches have already been used to estimate disease prevalence in the absence of a gold standard diagnostic test or when the gold standard has imperfect sensitivity and specificity [68]. The current review has identified gaps in the information required for mathematical models designed to accurately estimate prevalence of $P$. vivax infection. Health planning and decision making by malaria control and elimination programmes in $P$. vivax endemic areas will require reliable estimates of parasite prevalence. The results of this review highlight the benefits of investing in PCR techniques to inform malaria control programmes. In areas focused on elimination it is vital that all reservoirs of $P$. vivax malaria are detected especially since rates of asymptomatic carriage can be substantial with a known ability to transmit [69].

\section{Additional files}

Additional file 1. Studies excluded following evaluation of full text $(\mathrm{N}=142)$ according to the main reason for exclusion.

Additional file 2. Summary of study characteristics.

Additional file 3. Sensitivity and specificity of microscopy compared to PCR.

\section{Authors' contributions}

CM, MA and CD conceived of the study and developed the study design. CM searched the literature, extracted and analysed the data and prepared the manuscript. RP, MA and CD advised on the systematic review and meta-analysis. All authors read and approved the final manuscript.

\section{Author details}

${ }^{1}$ WorldWide Antimalarial Resistance Network (WWARN), Oxford, UK. ${ }^{2}$ Nuffield Department of Medicine, Centre for Tropical Medicine and Global Health, Oxford University, Oxford, UK. ${ }^{3}$ Faculty of Epidemiology and Population Health, London School of Hygiene and Tropical Medicine, University of London, London, UK. ${ }^{4}$ Gobal and Tropical Health Division, Menzies School of Health Research, Charles Darwin University, Darwin, Australia. ${ }^{5}$ Department of Immunology and Infection, London School of Hygiene and Tropical Medicine, London, UK. 


\section{Compliance with ethical guidelines}

\section{Competing interests}

The authors declare they have no competing interests.

Received: 30 June 2015 Accepted: 2 September 2015

Published online: 22 September 2015

\section{References}

1. Guerra CA, Howes RE, Patil AP, Gething PW, Van Boeckel TP, Temperley $\mathrm{WH}$, et al. The international limits and population at risk of Plasmodium vivax transmission in 2009. PLoS Negl Trop Dis. 2010;4:11.

2. Barcus MJ, Basri H, Picarima H, Manyakori C, Sekartuti, Elyazar l, et al. Demographic risk factors for severe and fatal vivax and falciparum malaria among hospital admissions in northeastern Indonesian Papua. Am J Trop Med Hyg. 2007;77:984-91.

3. Looareesuwan S, White N, Chittamas S, Bunnag D, Harinasuta T. High rate of Plasmodium vivax following treatment of falciparum malaria in Thailand. Lancet. 1987;7:1052-5.

4. Feachem $\mathrm{R}$, Sabot O. A new global malaria eradication strategy. Lancet. 2008;371:1633-5.

5. WHO. World malaria report 2008. Geneva: World Health Organization; 2008.

6. WHO. Guidelines for the diagnosis and treatment of malaria in the African region 2003. Geneva: World Health Organization; 2003.

7. Coura JR, Suárez-Mutis M, Ladeia-Andrade S. A new challenge for malaria control in Brazil: asymptomatic Plasmodium infection—a review. Mem Inst Oswaldo Cruz. 2006;101:229-37.

8. Roshanravan B, Kari E, Gilman RH, Cabrera L, Lee E, Metcalfe J, et al. Endemic malaria in the Peruvian Amazon region of lquitos. Am J Trop Med Hyg. 2003;69:45-52.

9. Snounou G, Viriyakosol S, Zhu XP, Jarra W, Pinheiro L, do Rosario VE, et al. High sensitivity of detection of human malaria parasites by the use of nested polymerase chain reaction. Mol Biochem Parasitol. 1993:61:315-20.

10. Carter R, Mendis KN. Evolutionary and historical aspects of the burden of malaria. Clinic. 2002;15:564-94.

11. Bousema T, Dinglasan RR, Morlais I, Gouagna LC, van Warmerdam T, Awono-Ambene $\mathrm{PH}$, et al. Mosquito feeding assays to determine the infectiousness of naturally infected Plasmodium falciparum gametocyte carriers. PLoS One. 2012;7:e42821.

12. Pourmoshtagh $\mathrm{H}$, Fahimzah $\mathrm{A}$, Karimi A. Comparison of polymerase chain reaction (PCR) and peripheral blood smear (PBS) for diagnosis of asymptomatic malaria parasitemia. Iran J Clin Infect Dis. 2012;7:7-9.

13. Okell LC, Ghani AC, Lyons E, Drakeley CJ. Submicroscopic infection in Plasmodium falciparum - endemic populations : a systematic review and meta-analysis. J Infect Dis. 2009;200:1509-17.

14. Cheng Q, Cunningham J, Gatton ML. Systematic review of sub-microscopic P. vivax infections: prevalence and determining factors. PLoS Negl Trop Dis. 2015;9:e3413.

15. Moher D, Liberati A, Tetzlaff J, Altman DG. Preferred reporting items for systematic reviews and meta-analyses: the PRISMA statement. PLoS Med. 2009;6:e1000097.

16. Harris RJ, Bradburn MJ, Deeks JJ, Hardbord RM, Altman DG, Sterne J. Metan: fixed-and random-effects meta-analysis. Stata J. 2008:8:3-28.

17. Alves FP, Durlacher RR, Menezes MJ, Krieger H, Silva LHP, Camargo EP. High prevalence of asymptomatic Plasmodium vivax and Plasmodium falciparum infections in native Amazonian populations. Am J Trop Med Hyg. 2002;66:641-8

18. Asih PB, Rozi IE, Herdiana Pratama NR, Hidayati AP, Marantina SS, et al. The baseline distribution of malaria in the initial phase of elimination in Sabang Municipality, Aceh Province, Indonesia. Malar J. 2012;11:291.

19. Barbosa S, Gozze AB, Lima NF, Batista CL, Bastos MDS, Nicolete VC, et al. Epidemiology of disappearing Plasmodium vivax malaria: a case study in rural Amazonia. PLoS Negl Trop Dis. 2014;8:e3109.

20. Cerutti C, Boulos M, Coutinho AF, Hatab MD, Falqueto A, Rezende HR, et al. Epidemiologic aspects of the malaria transmission cycle in an area of very low incidence in Brazil. Malar J. 2007;6:33.
21. Coleman RE, Sattabongkot J, Promstaporm S, Maneechai N, Tippayachai $\mathrm{B}$, Kengluecha A, et al. Comparison of PCR and microscopy for the detection of asymptomatic malaria in a Plasmodium falciparum/vivax endemic area in Thailand. Malar J. 2006:5:121.

22. Cole-tobian JL, Corte A, Baisor M, Kastens W, Xainli J, Bockarie M, et al. Age-acquired immunity to a Plasmodium vivax invasion ligand, the Duffy binding protein. J Infect Dis. 2002;186:531-9.

23. Congpuong K, Saejeng A, Sug-Aram R, Aruncharus S, Darakapong A, Meshnick SR, et al. Mass blood survey for malaria: pooling and real-time PCR combined with expert microscopy in north-west Thailand. Malar J. 2012;11:288.

24. Da Silva NS, da Silva-Nunes M, Malafronte RS, Menezes MJ, D’Arcadia RR, Komatsu NT, et al. Epidemiology and control of frontier malaria in Brazil: lessons from community-based studies in rural Amazonia. Trans R Soc Trop Med Hyg. 2010;104:343-50.

25. Fançony C, Gamboa D, Sebastião Y, Hallett R, Sutherland C, Sousa-Figueiredo JC, et al. Various pfcrt and pfmdr1 genotypes of Plasmodium falciparum cocirculate with $P$. malariae, $P$. ovale spp., and $P$. vivax in northern Angola. Antimicrob Agents Chemother. 2012;56:5271-7.

26. Gunasekera AW, Abeyasinghe RR, Premawansa S, Fernando SD. Usefulness of polymerase chain reaction to supplement field microscopy in a pre-selected population with a high probability of malaria infections. Am J Trop Med Hyg. 2011;85:6-11.

27. Kaisar MM, Supali T, Wiria AE, Hamid F, Wammes LJ, Sartono E, et al. Epidemiology of Plasmodium infections in Flores Island, Indonesia using real-time PCR. Malar J. 2013;12:169.

28. Kaneko A, Chaves LF, Taleo G, Kalkoa M, Isozumi R, Wickremasinghe $R$, et al. Characteristic age distribution of Plasmodium vivax infections after malaria elimination on Aneityum Island, Vanuatu. Infect Immun. 2014;82:243-52.

29. Kano FS, Sanchez BA, Sousa TN, Tang ML, Saliba J, Oliveira FM, et al. Plasmodium vivax Duffy binding protein: baseline antibody responses and parasite polymorphisms in a well-consolidated settlement of the Amazon Region. Trop Med Int Health. 2012;17:989-1000.

30. Kasehagen LJ, Mueller I, McNamara DT, Bockarie MJ, Rare L, Lorry K, et al. Changing patterns of Plasmodium blood-stage infections in the Wosera region of Papua New Guinea monitored by light microscopy and high throughput PCR diganosis. Am J Trop Med Hyg. 2013;75:588-96.

31. Katsuragawa TH, Gil LH, Tada MS, de Almeida e Silva A, Costa JD, Araújo Mda S, et al. The dynamics of transmission and spatial distribution of malaria in riverside areas of Porto Velho, Rondônia, in the Amazon region of Brazil. PLoS one. 2010;5:e9245.

32. Khaminsou N, Kritpetcharat O, Daduang J, Kritpetcharat P. A survey of malarial infection in endemic areas of Savannakhet province, Lao PDR and comparative diagnostic efficiencies of Giemsa staining, acridine orange staining, and semi-nested multiplex PCR. Parasitol Int. 2008;57:143-9.

33. Ladeia-Andrade S, Ferreira MU, de Carvalho ME, Curado I, Coura JR. Age-dependent acquisition of protective immunity to malaria in riverine populations of the Amazon Basin of Brazil. Am J Trop Med Hyg. 2009:80:452-9.

34. Li P, Zhao Z, Wang Y, Xing H, Parker DM, Yang Z, et al. Nested PCR detection of malaria directly using blood filter paper samples from epidemiological surveys. Malar J. 2014;13:175.

35. Lin E, Kiniboro B, Gray L, Dobbie S, Robinson L, Stanisic D, et al. Differential patterns of infection and disease with P. falciparum and $P$. vivax in young Papua New Guinean children. PLoS One. 2010;5:e9047.

36. Mehlotra RK, Kasehagen LJ, Baisor M, Lorry K, Kazura JW, Bockarie MJ, et al. Malaria infections are randomly distributed in diverse holoendemic areas of Papua New Guinea. Am J Trop Med Hyg. 2002;67:555-62.

37. Mehlotra RK, Lorry K, Kastens W, Miller SM, Alpers MP, Bockarie M, et al. Random distribution of mixed species malaria infections in Papua New Guinea. Am J Trop Med Hyg. 2000;62:225-31.

38. Mueller I, Widmer S, Michel D, Maraga S, McNamara DT, Kiniboro B, et al. High sensitivity detection of Plasmodium species reveals positive correlations between infections of different species, shifts in age distribution and reduced local variation in Papua New Guinea. Malar J. 2009;8:41.

39. Van Nguyen $H$, van den Eede $P$, van Overmeir $C$, Thang ND, Hung LX, D'Alessandro $U$, et al. Marked age-dependent prevalence of symptomatic and patent infections and complexity of distribution of human Plasmodium species in central Vietnam. Am J Trop Med Hyg. 2012;87:989-95. 
40. Patel JC, Lucchi NW, Srivastava P, Lin JT, Sug-Aram R, Aruncharus S, et al. Field evaluation of a real-time fluorescence loop-mediated isothermal amplification assay, RealAmp, for the diagnosis of malaria in Thailand and India. J Infect Dis. 2014;210:1180-7.

41. Rodulfo H, De Donato M, Mora R, Gonzalez L, Contreras CE. Comparison of the diagnosis of malaria by microscopy, immunochromatography and PCR in endemic areas of Venezuela. Braz J Med Biomed Res. 2007;40:535-43.

42. Rogawski ET, Congpuong K, Sudathip P, Satimai W, Sug-aram R, Aruncharus S, et al. Active case detection with pooled real-time PCR to eliminate malaria in Trat province, Thailand. Am J Trop Med Hyg. 2012;86:789-91.

43. Rosanas-Urgell A, Mueller D, Betuela I, Barnadas C, Iga J, Zimmerman P, et al. Comparison of diagnostic methods for the detection and quantification of the four sympatric Plasmodium species in field samples from Papua New Guinea. Malar J. 2010;9:361.

44. Rosas-Aguirre A, Llanos-Cuentas A, Speybroeck N, Cook J, Contreras-Mancilla J, Soto $V$, et al. Assessing malaria transmission in a low endemicity area of north-western Peru. Malar J. 2013;12:339.

45. Rubio JM, Benito A, Roche J, Berzosa PJ, García ML, Micó M, et al. Seminested, multiplex polymerase chain reaction for detection of human malaria parasites and evidence of Plasmodium vivax infection in Equatorial Guinea. Am J Trop Med Hyg. 1999;60:183-7.

46. Scopel KKG, Fontes CJF, Nunes ÁC, Horta MF, Braga ÉM. High prevalence of Plamodium malariae infections in a Brazilian Amazon endemic area (Apiacás-Mato Grosso State) as detected by polymerase chain reaction. Acta Trop. 2004;90:61-4

47. Singh B, Cox-Singh J, Miller AO, Abdullah MS, Snounou G. Detection of malaria in Malaysia by nested polymerase amplification of dried blood spots on filter papers chain reaction. Trans R Soc Trop Med Hyg. 1996;90:519-21.

48. Souza CRT, Carvalho T, Amaral RCG, Cunha LS, Cunha MG, Guerreiro JF. Prevalence of Plasmodium falciparum and P. vivax in an area of transmission located in Pará State, Brazil, determined by amplification of mtDNA using a real-time PCR assay. Genet Mol Res. 2012;11:3409-13.

49. Starzengruber P, Fuehrer H, Ley B, Thriemer K, Swoboda P, Habler VE, et al. High prevalence of asymptomatic malaria in South-Eastern Bangladesh. Malar J. 2014;13:1-10.

50. Steenkeste N, Incardona S, Chy S, Duval L, Ekala M-T, Lim P, et al. Towards high-throughput molecular detection of Plasmodium: new approaches and molecular markers. Malar J. 2009;8:86.

51. Suárez-mutis MC, Cuervo P, Leoratti FMS, Moraes-avila SL, Ferreira AW. Plasmodium vivax infection in the Amazon Rio Negro Area, Brazil. Rev Inst Med Trop Sao Paulo. 2007:49:159-64.

52. Versiani FG, Almeida MEM, Melo GC, Versiani FOL, Orlandi PP, Mariúba LAM, et al. High levels of IgG3 anti ICB2-5 in Plasmodium vivax-infected individuals who did not develop symptoms. Malar J. 2013;12:294.

53. Vo TKD, Bigot P, Gazin P, Sinou V, De Pina JJ, Huynh DC, et al. Evaluation of a real-time $P C R$ assay for malaria diagnosis in patients from Vietnam and in returned travellers. Trans R Soc Trop Med Hyg. 2007;101:422-8.

54. Wang B, Han S-S, Cho C, Han J-H, Cheng Y, Lee S-K, et al. Comparison of microscopy, nested-PCR, and real-time-PCR assays using high-throughput screening of pooled samples for diagnosis of malaria in asymptomatic carriers from areas of endemicity in Myanmar. J Clin Microbiol. 2014;52:1838-45.
55. De Almeida A, Rosário VE, Henriques G, Arez AP, Cravo P. Plasmodium vivax in the Democratic Republic of East Timor: parasite prevalence and antifolate resistance-associated mutations. Acta Trop. 2010;115:288-92.

56. Gomes ADC, Paula MB De, Duarte AMRDC, Lima MA, Malafronte RDS, Mucci LF, Gotlieb SLD, Natal D. Epidemiological and ecological aspects related to malaria in the area of influence of the lake at Porto Primavera dam, in western São Paulo State, Brazil. Rev Inst Med Trop Sao Paulo. 2008;50:287-95.

57. Das MK, Joshi H, Verma A, Singh SS, Adak T. Malaria among the Jarawas, a primitive and isolated tribe on the Andaman islands, India. Ann Trop Med Parasitol. 2005;99:545-52.

58. Turki H, Zoghi S, Mehrizi AA, Zakeri S, Raeisi A, Khazan H, Haghdoost A. Absence of asymptomatic malaria infection in endemic area of bashagard district, hormozgan province, iran. Iran J Parasitol. 2012;7:36-44.

59. Zoghi S, Mehrizi AA, Raeisi A, Haghdoost AA, Turki H, Safari R, Kahanali AA, Zakeri S: Survey for asymptomatic malaria cases in low transmission settings of Iran under elimination programme. Malar J. 2012;11:126.

60. WHO. Basic malaria microscopy part 1: learner's guide 1991. Geneva: World Health Organization; 1991.

61. Kasehagen LJ, Mueller I, McNamara DT, Bockarie MJ, Kiniboro B, Rare L, et al. Changing patterns of Plasmodium blood-stage infections in the Wosera region of Papua New Guinea monitored by light microscopy and high throughput PCR diagnosis. Am J Trop Med Hyg. 2006;75:588-96.

62. Mcnamara DT, Cole-Tobian J. Diagnosing infection levels of four human malaria parasite species by a polymerase chain reaction/microspherebased assay. Am J Trop Med Hyg. 2013;74:413-21.

63. Taylor S, Juliano J, Trottman P, Griffin J, Landis S, Kitsa P, et al. Highthroughput pooling and real-time PCR-based strategy for malaria detection. J Clin Microbiol. 2010;48:512-9.

64. Snounou G, Viriyakosol S, Jarra W, Thaithong S, Brown K. Identification of the four human malaria parasite species in field samples by the polymerase chain reaction and detection of a high prevalence of mixed infections. Mol Biochem Parasitol. 1993;58:283-92.

65. Mbakilwa H, Manga C, Kibona S, Mtei F, Meta J, Shoo A, et al. Quality of malaria microscopy in 12 district hospital laboratories in Tanzania. Pathog Glob Health. 2012;106:330-4.

66. Meneghini R, Packer AL. Is there science beyond English? EMBO Rep. 2007;8:2-6.

67. Babiker H, Schneider P, Reece S. Gametocytes: insights gained during a decade of molecular monitoring. Trends Parasitol. 2008;24:525-30.

68. Lu Y, Dendukuri N, Schiller I, Joseph L. A Bayesian approach to simultaneously adjusting for verification and reference standard bias in diagnostic test studies. Stat Med. 2010;29:2532-43.

69. Alves FP, Gil L, Marrelli MT, Ribolla PE, Camargo EP, Da Silva LH. Asymptomatic carriers of Plasmodium spp. as infection source for malaria vector mosquitoes in the Brazilian Amazon. J Med Entomol. 2005;42:777-9.

\section{Submit your next manuscript to BioMed Central and take full advantage of:}

- Convenient online submission

- Thorough peer review

- No space constraints or color figure charges

- Immediate publication on acceptance

- Inclusion in PubMed, CAS, Scopus and Google Scholar

- Research which is freely available for redistribution

Submit your manuscript at

www.biomedcentral.com/submit

C BioMed Central 